Cuadernos de Gobierno y Administración Pública

ISSN: e-2341-4839

http://dx.doi.org/10.5209/CGAP.57915

\title{
Colaboración en Colombia. El caso del Consejo Nacional de Planeación
}

\author{
Camila Alvarado Jaimes*
}

Recibido: 31/08/2017 / Aceptado: 9/10/2017

Resumen. El objetivo de este artículo es analizar el Consejo Nacional de Planeación (CNP), principal instancia en Colombia de concertación entre el gobierno y actores no estatales, civiles y económicos, en los procesos de planificación del país. Se busca evaluar, entonces, la autonomía del Consejo y su diseño institucional y realizar un análisis crítico a partir de dos marcos de referencia: la aspiración constitucional que estableció su creación y la orientación teórica de la gobernanza moderna. De acuerdo con el carácter descriptivoanalítico de la investigación, se determinó como herramienta metodológica el análisis documental, en tanto facilitó el acceso a la información y proporcionó un modelo intelectual para su recuperación y análisis.

Palabras clave: Colaboración, gobernanza, planeación participativa, decisión pública.

\section{Collaboration in Colombia. The case of the National Planning Council}

\begin{abstract}
The objective of this article is to analyze the National Planning Counsel (NPC), main institution in Colombia for the coordination between the government and non-state actors, such as civil and economic actors, for the planning processes in the country. The intention is to evaluate the autonomy of the Counsel and its institutional design and elaborate a critical analyzes taking into account two frame works: the purpose of its constitutional origin and the orientation given by the modern governance theory. According to the descriptive-analytic character of the investigation, document analysis was determined as the methodological instrument, as much as it facilitated the access to the information and it provided an intellectual model for its analysis and recovery.
\end{abstract}

Keywords: Collaboration, governance, participatory planning, public decision.

Sumario. 1. Introducción. 2.Marco teórico-conceptual: Gobernanza moderna. 3. Antecedentes y configuración institucional del CNP. 3.1. Marco Jurídico del CNP. 3.2. De las disposiciones normativas del CNP: Funciones, miembros y alcances. 4. Análisis crítico del CNP. 4.1. Aspiración constitucional vs. Realidad del CNP. 4.2. Revisión crítica del CNP desde la perspectiva de la gobernanza moderna. 5. Conclusiones. Bibliografía.

Cómo citar: Alvarado Jaimes, C., «Colaboración en Colombia. El caso del Consejo Nacional de Planeación», en Cuadernos de Gobierno y Administración Pública 4-2, 113-133.

\section{Introducción}

En Colombia, los esquemas de colaboración público-privado como instrumento al servicio del desarrollo del país se han incorporado a la gestión de lo público en dos

* Universidad del Rosario. 
líneas con ritmos de evolución diferentes. En primer lugar, instancias cuyo campo de acción son los procesos de decisión pública y el diseño de estrategias de desarrollo vía los mecanismos de planificación del país y más adelante, en una segunda línea, los esquemas de trabajo afiliados a la nueva gestión pública (NGP) que vinculan al sector privado con la externalización de funciones públicas y en la ejecución de políticas.

A partir de la década de 1960, en Colombia se introdujo formalmente la planeación como parte de las tareas de gobierno. Este ejercicio de planificación se realiza en función de los ciclos políticos y su objetivo es establecer prioridades estratégicas para el desarrollo económico y la transformación productiva del país a mediano y largo plazo.

Pese a que en muchos casos ha sido reducido a un instrumento de justificación del gasto público, la planificación fue el marco original de diálogo entre las entidades de gobierno y los principales actores económicos y organizaciones sociales. A partir de aquí, han existido un sinnúmero de iniciativas orientadas a crear sinergias y mecanismos de trabajo coordinado para la solución efectiva de problemas públicos.

Representantes de sector empresarial y organizaciones sociales, participan en la producción de estrategias a través de mesas de diálogo, comisiones especiales e, incluso, órganos permanentes asesores de la Presidencia para la formulación de planes de desarrollo nacionales y regionales.

Actualmente, en Colombia coexisten múltiples instancias de colaboración público-privada que van desde alianzas de tipo formal y estructurado hasta diálogos tácitos o informales convocados por el gobierno en torno a alcanzar entendimiento y consenso sobre algún asunto particular.

Sin embargo, en los últimos años ha habido una tendencia a formalizar estas estructuras de diálogo, lo cual ha permitido alcanzar importantes consensos, impulsar procesos creativos en la solución de problemas, definir estrategias, fortalecer la presencia institucional del Estado en zonas de conflicto a través de actores regionales e, incluso, alcanzar entendimiento con sectores que representan visiones estratégicas del desarrollo distintas a la visión del gobierno.

Así, entonces, a partir de la constituyente de 1991 y en las décadas posteriores, se ha legislado, otorgando un estatus formal a este tipo de diálogos para asegurar la legitimación de las alianzas y protegerlas de las diferentes concepciones que vienen con cada nuevo gobierno. No obstante, existen algunos casos de diálogos estructurados informales, que se expresan en consejos o comisiones, que fueron adquiriendo legitimidad de hecho e institucionalizándose.

De aquí, que la proliferación de formas y vínculos entre el gobierno y actores no estatales para trazar objetivos comunes sobre la base de la acción colectiva y coordinada, promueva la formalización de estructuras de diálogo a nivel nacional y regional con el objeto de lograr consenso y aunar recursos en torno a las líneas de desarrollo que debe seguir el país.

Si bien, los diferentes esquemas de trabajo entre el sector público y privado -haciendo referencia a las instancias que intervienen los procesos de decisión pública y aquellas que se vinculan con la gestión y ejecución de políticas- han contribuido a difundir y favorecido nuevos modelos de gobernanza en el país, no todos contienen implícita la noción de colaboración ni conducen, necesariamente, a un nuevo modelo de dirección política distinto al enfáticamente jerárquico y centralista que ha imperado hasta ahora en Colombia. 
De aquí, que este artículo se proponga analizar el caso del Consejo Nacional de Planeación (CNP), instancia más importante de planificación participativa y única de colaboración entre el gobierno y actores no estatales creada por mandato constitucional, cuya aspiración original fue ampliar los centros de decisión pública a través de la vinculación de actores privados, económicos y sociales, en los procesos de planificación del país. Así, pues, en el CNP confluyen representantes de las entidades territoriales y de los distintos sectores que integran la sociedad civil colombiana con el objeto de intervenir las fases y los procesos de planificación del país en todos los niveles.

El análisis del CNP estará orientado por la perspectiva teórica de la gobernanza moderna, en tanto ofrece un marco explicativo acerca de las tensiones que ejercen las recientes transformaciones sociales y económicas sobre los sistemas democráticos e incorpora los imperativos de la colaboración entre actores públicos y privados como eje central de un nuevo modo de dirección de la sociedad. De acuerdo Aguilar (2005), "el corazón del concepto de gobernanza es un concepto descentralizado de la dirección de la sociedad que ya no puede ser dirigida por un centro" (Serrano, 2011: 5).

Así entonces, en una primera parte se presentarán las principales posturas teóricas que han conceptualizado alrededor de la gobernanza moderna o nueva gobernanza. Posteriormente, se estudiará el contexto que precedió y condujo al establecimiento del Consejo y se presentarán las disposiciones normativas que definen su organización, integrantes, alcance y funciones. Finalmente, se analizará la labor, consolidación y autonomía del Consejo desde dos aproximaciones: desde la perspectiva constitucional que dispuso su creación y desde la orientación teórica de la gobernanza moderna.

\section{Marco teórico-conceptual: Gobernanza moderna}

El análisis del CNP como espacio de concertación entre el gobierno y actores no estatales, estará dirigido por la perspectiva teórica de la gobernanza moderna.Pese a que el concepto de gobernanza no es nuevo, su uso durante mucho tiempo hizo referencia al mero proceso de "gobernar".Es por esto, que la nueva literatura académica apela al término nueva gobernanza o gobernanza moderna con el objeto de separarlo conceptualmente del término anterior y para indicar un nuevo modo de gobernar.

El debate académico sobre la gobernanza moderna desarrollado fuertemente a partir de la década de los noventa, intenta dar cuenta de las transformaciones del Estado en las décadas anteriores. El fracaso parcial de las políticas de reforma y reconstrucción de los Estados del occidente de Europa después de concluir la Segunda Guerra Mundial y la desaceleración del crecimiento económico en la década de los setenta, cuestionaron la capacidad del Estado para asegurar un bienestar permanentemente creciente y su rol como centro efectivo de la dirección de la sociedad.

De aquí, que se evaluaran nuevas vías para guiar el desarrollo socio-económico y se diera paso al mercado para dirigir la transformación política y económica a través de una serie de políticas de orden neoliberal, orientadas hacia la desregulación, privatización, menor intervención del Estado e inserción competitiva de las economías en el mercado mundial.

Así mismo, en América Latina la crisis de deuda de los Estados en la década de 1980, impuso una serie de presiones en la región por parte de organismos interna- 
cionales que condujeron a un proceso de adopción de estas políticas de liberalización económica.

Si bien, la idea del mercado como fuerza de desarrollo integral ha sido fuertemente desacreditada por una serie de contratiempos políticos y económicos, ha producido un efecto importante en la forma de entender la gestión de lo público y la relación entre los diferentes actores de la sociedad.

En términos generales, las transformaciones políticas, económicas y sociológicas del siglo XX y que siguen en curso, se traducen en una pérdida de capacidad del gobierno para dirigir el desarrollo de una sociedad y conducir su crecimiento económico, en tanto han reducido sus poderes, recursos y facultades para intervenir los asuntos públicos (Aguilar, 2007).

Este factor de insuficiencia del gobierno expuesto en las décadas de 1980 y 1990 , reveló la necesidad de un nuevo modelo de dirección política de la sociedad con el objeto de superar las restricciones del Estado y "ascender a un nivel superior de información, inteligencia, recursos, organización y eficiencia que rebasa las potencialidades gubernamentales, razón por la cual se exigen formas sinérgicas de deliberación, interacción y asociación público-privada" (Aguilar, 2007: 8).

De aquí, que la gobernanza moderna haya adquirido mayor atención como modelo alternativo al Estado jerárquico tradicional y a la hegemonía del mercado en la regulación de la sociedad y la gestión de lo público.

El concepto de gobernanza, en principio, fue un concepto descriptivo, el cual fue reelaborado en concepto teórico en la década de 1990, bajo la obra liderada por Jan Kooiman (1993): Modern Governance: New Government, Society Interactions, la cual estimuló una serie de estudios académicos y conceptualizaciones por distintos autores.

Entre ellos, Bob Jessop (1998) afirma que la gobernanza pretende dar cuenta de las transformaciones del papel del gobierno en un contexto de globalización, relocalización, complejidad social, descentramiento de la política y pérdida del carácter autosuficiente del Estado y es definida como la interdependencia y coordinación negociada entre sistemas y organizaciones.

Renate Mayntz señala que la gobernanza moderna significa una "forma de gobernar más cooperativa, diferente del antiguo modelo jerárquico, en el que las autoridades estatales ejercían un poder soberano sobre los grupos y ciudadanos que constituían la sociedad civil" (Mayntz, 2001: 1). En la gobernanza moderna, las instituciones estatales y no estatales, los actores públicos y privados, participan y a menudo cooperan en la formulación y aplicación de políticas públicas.

En la misma línea, Cristina Zurbriggen (2011: 1) afirma que "[...] la gobernanza surge como un nuevo estilo de gobierno distinto del modelo de control jerárquico y de mercado, caracterizado por un mayor grado de cooperación entre los gobiernos y administraciones públicas y actores no gubernamentales en la hechura de políticas públicas". Así, pues, este proceso de elaboración de las políticas públicas fundamentado en la colaboración, el consenso y la participación de distintos actores, mejora el rendimiento de las políticas y, en consecuencia, garantiza la gobernabilidad del sistema político.

De acuerdo con Rhodes, principal exponente de la escuela anglosajona de gobernanza, la estructura de la gobernanza moderna no se caracteriza por la jerarquía, sino por actores corporativos autónomos y por redes entre organizaciones (Rhodes, 1997). Rhodes centra el desarrollo académico de la gobernanza en el concepto de redes de políticas públicas o policy networks, intentando comprender las diferentes 
modalidades de interacción entre los intereses de actores públicos y privados en la formulación e implementación de políticas públicas.

Siguiendo a Aguilar, se entiende por gobernanza moderna "al proceso en el que la definición del sentido de dirección de la sociedad, de las formas de organizarse para realizar los objetivos -resolver problemas, enfrentar desafíos, crear futuros de valía- y del modo como se distribuirán los costos y beneficios ya no puede ser obra exclusiva del gobierno, considerado como el actor único o el dominante, sino que es el resultado de la deliberación conjunta, interacción, interdependencia, coproducción, corresponsabilidad, asociación entre el gobierno y las organizaciones privadas y sociales" (Aguilar, 2007: 10).

De aquí, que la gobernanza moderna como proceso de gobierno o dirección de la sociedad implique un proceso deliberativo y colaborativo entre el gobierno y actores no estatales -empresas privadas y organizaciones de la sociedad civil-, en los procesos de decisión pública, establecimiento de objetivos y estrategias de desarrollo.

Si bien existen distintos estudios y enfoques de investigación acerca del concepto de gobernanza, entre ellos, quienes se centran en las transformaciones del rol del Estado y de sus funciones administrativas y aquellos cuya orientación teórica hace mayor énfasis en el estudio redes de políticas públicas; este artículo se vinculará con la perspectiva académica que centra su atención en la gobernanza como un nuevo modo de gestionar los asuntos públicos fundamentado en un mayor grado de cooperación entre el gobierno y actores no estatales en procesos de decisión pública y diseño de políticas públicas, haciendo énfasis en la dimensión de acción colectiva entre el gobierno y sectores no estatales.

Desde esta perspectiva, la asociación y colaboración público-privada se entiende como instrumento de reproducción de la gobernanza moderna, en tanto implica la acción colectiva entre el gobierno y actores no estales, práctica que ha contribuido a difundir y normalizar la nueva gobernanza como modelo de gobierno y forma de dirigir el desarrollo de las sociedades modernas.

\section{Antecedentes y configuración institucional del CNP}

En 1991, la Asamblea Nacional Constituyente, introdujo como mandato constitucional instancias de concertación y participación de actores no estatales, con el objetivo de corregir las desviaciones y problemas que se presentaron durante la vigencia de la Constitución de 1886, que coartaron las visiones y propuestas políticas distintas a las impuestas por la élite política del centro del país.

Debido a lo anterior y los conflictos consecuentes en la periferia del país, la Carta de 1991 estableció en su segundo artículo como uno de los fines esenciales del Estado colombiano "[...] facilitar la participación de todos en las decisiones que los afectan y en la vida económica, política, administrativa y cultural de la Nación" (Constitución Política de Colombia, 1991).

Para hacer efectivo el mandato constitucional, las instituciones del Estado colombiano, responsables dirigir los procesos de decisión pública y de diseñar políticas, deben asegurar espacios de concertación y participación de sectores privados, económicos y sociales, que conforman la sociedad civil colombiana, en los procesos de planeación y producción de estrategias de desarrollo. 
Así mismo, constitucionalmente se estableció que los procesos de planificación del país tendrían un carácter indicativo para la sociedad y obligatorio para el Estado y se contempló, desde su concepción, la vinculación y colaboración de actores no gubernamentales a través de la creación del Sistema Nacional de Planeación, conformado por los consejos de planeación, nacionales y territoriales.

Así, entonces, con el propósito de incorporar actores no estatales tradicionalmente excluidos en los procesos de planeación y en el debate de políticas públicas, se estableció la creación del Consejo Nacional de Planeación, integrado por representantes de entidades territoriales y de los sectores económicos, sociales, ecológicos, comunitarios y culturales y de los Consejos Territoriales de Planeación (CTP), los cuales constituyen el Sistema Nacional de Planeación. ${ }^{1}$

El CNP, única instancia de colaboración entre el gobierno y actores no estatales de origen constitucional, modificó, al menos desde lo formal, el curso de los procesos de planeación en Colombia cuya esencia previa a los consejos "se traducía en reuniones a puerta cerrada de expertos con dones para leer desde allí las realidades del país, interpretarlas, problematizarlas y promover cursos de acción para resolver los problemas sociales [del país]" (Puentes y Gonzáles, 2011: 205).

Las palabras del ex magistrado Ciro Angarita reflejan la lógica de la dirección política que se buscó revertir a través de la creación del CNP:

"La existencia de un pequeño grupo de gentes educadas en el centro del país o en el exterior, reunidas en el Departamento Nacional de Planeación y otras instituciones técnicas de orden nacional, orientadas por una ideología estatista, justificaba el trato de las regiones como menores de edad. Las instituciones políticas de esa época, que no permiten ninguna decisión en la periferia, reflejan esa concepción [...], en síntesis, se suponía que un grupo selecto de personas podía escoger lo más conveniente para la Nación por encima de las regiones y del funcionamiento de los mercados" (Angarita, 1995. En: Puentes y Gonzáles, 2011: 206).

Así, entonces, la asamblea nacional constituyente buscó institucionalizar la colaboración entre representantes de la sociedad civil y de gremios económicos y el gobierno, creando un foro para la discusión del Plan Nacional de Desarrollo, ${ }^{2}$ a nivel

1 Artículo 340, Constitución Política de Colombia: "Habrá un Consejo Nacional de Planeación, integrado por representantes de entidades territoriales y de los sectores económicos, sociales, ecológicos, comunitarios y culturales. El Consejo tendrá carácter consultivo y servirá de foro para la discusión del Plan Nacional de Desarrollo. Los miembros del Consejo Nacional serán designados por el presidente de la República de listas que le presenten las autoridades y las organizaciones de las entidades y sectores a que se refiere el inciso anterior, quienes deberán estar o haber estado vinculados a dichas actividades. Su período será de ocho años y cada cuatro se renovará parcialmente en la forma que establezca la ley. En las entidades territoriales habrá también consejos de planeación, según lo determine la ley. El Consejo Nacional de Planeación y los consejos territoriales de planeación constituyen el Sistema Nacional de Planeación”.

2 Artículo 339, Constitución política de Colombia (1991). "Habrá un Plan Nacional de Desarrollo conformado por una parte general y un plan de inversiones de las entidades públicas del orden nacional. En la parte general se señalarán los propósitos y objetivos nacionales de largo plazo, las metas y prioridades de la acción estatal a mediano plazo y las estrategias y orientaciones generales de la política económica, social y ambiental que serán adoptadas por el gobierno. El plan de inversiones públicas contendrá los presupuestos plurianuales de los principales programas y proyectos de inversión pública nacional y la especificación de los recursos financieros requeridos para su ejecución. Las entidades territoriales elaborarán y adoptarán de manera concertada entre ellas y el gobierno nacional, planes de desarrollo, con el objeto de asegurar el uso eficiente de sus recursos y el desempeño adecuado de las funciones que les hayan sido asignadas por la Constitución y la ley. Los planes de las entidades territoriales estarán conformados por una parte estratégica y un plan de inversiones de mediano y corto plazo." 
central y en los diferentes niveles territoriales -departamental y municipal-. Posteriormente, se determinó en el Estatuto Orgánico de Planeación (1994), ${ }^{3}$ que las máximas autoridades administrativas de las entidades territoriales tendrían al menos cuatro asientos en el CNP, con el objeto de servir de vínculo y coordinación entre los diferentes niveles de la administración pública.

\subsection{Marco jurídico del CNP}

El Consejo Nacional de Planeación fue creado por la carta constitucional de 1991 y posteriormente, reglamentado de acuerdo con el siguiente desarrollo normativo y jurisprudencial:

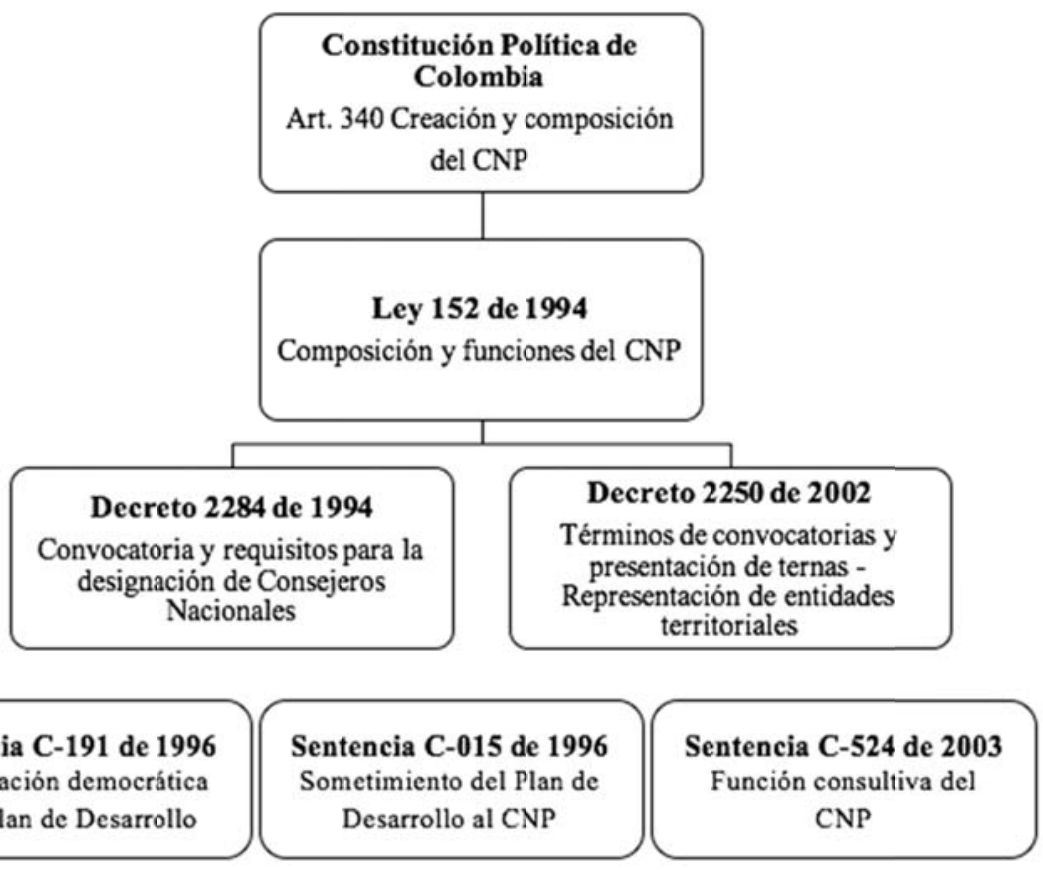

Fuente: Departamento Nacional de Planeación, 2017.

La creación del Consejo Nacional de Planeación es dictada por el Art. 340 de la Constitución Política de Colombia, el cual establece que el mismo estará integrado por representantes de las entidades territoriales y de los sectores económicos, sociales, ecológicos, comunitarios y culturales. Adicionalmente le otorga carácter consultivo y establece que su principal función será servir de foro para la discusión del Plan Nacional de Desarrollo.

La denominada Ley Orgánica del Plan de Desarrollo, ley 152 de 1994, estableció las autoridades e instancias de planeación, otorgándole al Consejo Nacional de Planeación, junto con el Congreso de la República, el carácter de instancia.

3 Véase: Ley 152 de 1994, conocida como el Estatuto Orgánico de Planeación. 
Adicionalmente establece su composición, las calidades de los integrantes del Consejo, su periodo, funciones y el proceso de designación por parte del presidente de la República.

Bajo el Decreto 2284 de 1994 se establece que la participación de los Consejeros Nacionales de Planeación, representantes de las entidades territoriales, se hará con independencia de la persona que ejerza los cargos de gobernador y alcalde, según sea el caso. Adicionalmente reglamenta los parámetros para el proceso de designación de los Consejeros Nacionales.

El proceso para la designación de los Consejeros Nacionales representantes de las entidades territoriales es establecido por el Decreto 2250 de 2002, proceso que se lleva a cabo según los grupos que establece esta norma, adicionalmente implementa el proceso de convocatoria adelantada por el Departamento Nacional de Planeación, así como la presentación de las ternas para la postulación.

Los aspectos relacionados con la designación de los Consejeros Nacionales de Planeación y con su naturaleza son contemplados por la Sentencia No. C-015/96. Respecto al concepto que debe emitir el CNP, la sentencia señala que: "es requisito indispensable, de obligatoria observancia, para la validez de la ley mediante la cual se adopte el Plan Nacional de Desarrollo".

Bajo la sentencia C-191/96 se establece que la participación democrática debe permear todo el proceso de planeación y no sólo la elaboración del Plan. Esto significa que es perfectamente legítimo que la ley establezca, dentro de ciertos límites, mecanismos de ejecución, fiscalización y evaluación del Plan que sean esencialmente participativos.

Así mismo, bajo la Sentencia C-524/03 se enuncia que "la realización del principio de participación consagrado en la Carta Política exige que la actuación del Consejo Nacional de Planeación y de los consejos territoriales, como instancias para la discusión del Plan de Desarrollo, se garantice no sólo en la fase de aprobación sino también frente a las modificaciones del Plan, lo que les otorga a dichos consejos permanencia institucional para el cumplimiento de su función consultiva". En consecuencia, la función consultiva de los Consejos de Planeación no se agota en la fase de discusión del Plan, sino que se extiende a las etapas subsiguientes relacionadas con su modificación.

A nivel municipal y departamental se dispone constitucionalmente la creación de instancias territoriales de planeación, Consejos Territoriales de Planeación (CTP), cuyo objetivo es garantizar la participación de actores de la sociedad civil en la construcción y seguimiento de políticas públicas a nivel territorial, en virtud del principio de la planeación participativa. A continuación, el desarrollo normativo que regula los CTP:

El art. 340 de la Constitución Política determinó que en las entidades territoriales también habría Consejos de Planeación, los cuales junto con el Consejo Nacional constituirían el Sistema Nacional de Planeación.

Así mismo, se establecen las autoridades e instancias territoriales de planeación por la Ley Orgánica del Plan de Desarrollo, otorgándole a los CTP junto con las Asambleas Departamentales, los Concejos Municipales, Distritales y de las Entidades Territoriales Indígenas, el carácter de instancia. Adicionalmente implementa su composición y funciones. 
Posteriormente, bajo el desarrollo jurisprudencial de la sentencia C-191/96 se determina que los procesos de elaboración, ejecución, seguimiento y evaluación de los planes de desarrollo, tanto a nivel nacional como a nivel de las entidades territoriales, deben ser, en lo posible, participativos, puesto que uno de los fines esenciales del Estado es "facilitar la participación de todos en las decisiones que los afectan y en la vida económica, política y administrativa de la Nación".

Finalmente, en el año 2013 la sentencia C-524/03 resalta el papel que deben tener los consejos en relación con los planes de desarrollo de las respectivas entidades territoriales. Establece que la función consultiva de los Consejos de Planeación no se agota en la fase de discusión del Plan, sino que se extiende a las etapas subsiguientes relacionadas con su modificación.

\subsection{De las disposiciones normativas del CNP: Funciones, miembros y alcances}

Por mandato constitucional se crea el Consejo Nacional de Planeación (CNP), otorgándole carácter consultivo y sirviendo de foro de diálogo y concertación entre actores públicos y privados para la construcción de políticas públicas y la formulación de los planes de desarrollo.

Como se mencionó anteriormente, la creación del CNP fue dispuesta por la Asamblea Constituyente de 1991, la cual dejó al Congreso de la República la labor de reglamentar e institucionalizar el acercamiento de los actores no gubernamentales a los centros de decisión del país.

Así las cosas, bajo el Estatuto Orgánico de Planeación (Artículo 12o, Ley 152 de 1994) se otorga al CNP el carácter de instancia nacional de planeación, se determinan los parámetros de su funcionamiento y se derivan como sus principales funciones:

- Analizar y discutir el proyecto del Plan Nacional de Desarrollo, lo cual implica organizar y coordinar una amplia discusión nacional sobre el proyecto del Plan Nacional de Desarrollo, mediante la organización de reuniones nacionales y regionales con los Consejos Territoriales de Planeación en las cuales intervengan los sectores económicos, sociales, ecológicos, comunitarios y culturales, con el fin de garantizar eficazmente la participación ciudadana de acuerdo con el artículo 342 de la Constitución Política.

- Absolver las consultas que, sobre el Plan Nacional de Desarrollo, formule el Gobierno Nacional o las demás autoridades de planeación durante la discusión del proyecto del plan; de esta forma, la función consultiva del Consejo Nacional de Planeación y de los Consejos Territoriales de Planeación no se agota en la fase de discusión del Plan de Desarrollo, sino que se extiende a las etapas subsiguientes en relación con la modificación de dichos planes. ${ }^{4}$

- Formular recomendaciones a las demás autoridades y organismos de planeación sobre el contenido y la forma del Plan.

- Conceptuar sobre el proyecto del Plan de Desarrollo elaborado por el gobierno.

Sin embargo, en el año 2003, las funciones del CNP fueron ampliadas por la Corte Constitucional (Sentencia C-524 de 2003), la cual especificó que la labor de

4 Texto subrayado declarado exequible por la Corte Constitucional, mediante Sentencia C-524 de 2003. 
los Consejos de Planeación incluye el seguimiento y evaluación de los planes de desarrollo. Para cumplir con su función de seguimiento y control sobre las estrategias de desarrollo que deben seguir los gobiernos, se han formado amplias redes de apoyo, integradas por ciudadanos y organizaciones sociales.

La instancia regional del CNP, los Consejos Territoriales de Planeación (CTP), son creados para asegurar la participación ciudadana en la construcción y seguimiento de políticas públicas a nivel territorial. Los CTP cumplen las mismas funciones orgánicas del CNP a nivel departamental y municipal.

De acuerdo con el Estatuto Orgánico de Planeación, el CNP es convocado por el Gobierno una vez el presidente ha tomado posesión de su cargo y está integrado por representantes de las entidades territoriales y de diferentes sectores de la sociedad civil, quienes son elegidos por el presidente de la República de ternas propuestas por las correspondientes autoridades y organizaciones.

La presentación de ternas, el proceso de convocatoria y la designación de los consejeros y consejeras Nacionales de Planeación y de representantes de las entidades territoriales, se reglamenta mediante decreto en 1994 y se establece que sólo organizaciones con personería jurídica podrán presentar ternas ante el Departamento Nacional de Planeación para la posterior selección y designación de los representantes y miembros del CNP por parte del presidente de la República. El nombramiento por parte del presidente se realiza a título personal.

Los sectores con representación en el Consejo por decreto, ${ }^{5}$ son el económico, social, educativo y cultural, ecológico, minorías étnicas, comunidades negras, islenas y organizaciones que representen las mujeres.

Con respecto al sector económico, las personas jurídicas que agremien y asocien a los industriales, los productos agrarios, los comerciantes, las entidades financieras y aseguradoras, los microempresarios y las empresas y entidades de prestación de servicios. Con respecto al sector social, las personas jurídicas que agremien o asocien a los profesionales, campesinos, empleados, obreros, trabajadores independientes e informales.

En representación del sector educativo y cultural, las agremiaciones nacionales jurídicamente reconocidas de las universidades, las organizaciones jurídicamente reconocidas que agrupen a nivel nacional instituciones de educación primaria y secundaria de carácter público o privado, las organizaciones nacionales legalmente constituidas cuyo objeto sea el desarrollo científico, técnico o cultural y las organizaciones que agrupen a nivel nacional los estudiantes universitarios.

Con referencia al sector ecológico, las organizaciones jurídicamente reconocidas cuyo objeto sea la protección y defensa de los recursos naturales y del medio ambiente. Del sector comunitario, por su parte, las agremiaciones nacionales de asociaciones comunitarias con personería jurídica.

Finalmente, como representantes de los indígenas y las minorías étnicas, las organizaciones nacionales jurídicamente reconocidas que agrupen a los indígenas, las comunidades negras y las comunidades isleñas raizales del Archipiélago de San Andrés, Providencia y Santa Catalina, y para los representantes de las mujeres, las organizaciones no gubernamentales cuyo objeto sea la protección y defensa de los derechos de la mujer.

Artículo 5º Decreto 2284 de 1994.- En los términos señalados por este Decreto, se establecen las organizaciones con personería jurídica con derecho a presentar ternas para la designación por el presidente de la República de los representantes correspondientes ante el Consejo Nacional de Planeación. 
Así las cosas, los integrantes de $\mathrm{CNP}^{6}$ son elegidos por un período de ocho años -el doble del período presidencial-, con el propósito de que la responsabilidad de mantener la continuidad de los planes de desarrollo repose en estos consejos. No obstante, se establece que cada cuatro años podrá realizarse una renovación parcial de los miembros de los distintos sectores representados en el Consejo.

Finalmente, la sostenibilidad financiera y orgánica del CNP descansa sobre DNP, reglamentándose que "el Departamento Nacional de Planeación prestará al Consejo el apoyo administrativo y logístico que sea indispensable para su funcionamiento"(Sentencia C-524 de 2003).

\section{Análisis crítico del CNP}

La Asamblea Nacional Constituyente de 1991 se planteó dar un giro a la realidad política del país, la nueva carta buscó fortalecer el proceso de democratización y reorientar el modelo de dirección política de la sociedad que hasta ahora había excluido visiones diferentes a las de las élites políticas centrales y generado un gran caos institucional.

Sin embargo, el marco valorativo e institucional colombiano ha restringido la participación de otros actores del marco democrático en el espacio de deliberación de los asuntos públicos. A continuación, entonces, se realizará un análisis crítico de la labor del Consejo desde la visión constitucional que dispuso su creación y desde la perspectiva teórica de la gobernanza moderna.

\subsection{Aspiración constitucional vs. Realidad del CNP}

El desarrollo normativo y jurisprudencial alrededor del Consejo ha definido criterios claros sobre los cuales descansa la labor del CNP, como lo refleja el siguiente apartado de la Sentencia C-015 de 1996 de la Corte Constitucional:

"Como una manifestación del principio básico de la democracia participativa, la Constitución Política ha hecho indispensable que, antes de pasar por el Congreso, el proyecto del Plan General de Desarrollo sea conocido, estudiado, discutido y evaluado por los distintos sectores de la sociedad, cuyo concepto debe ser tenido en cuenta por el Ejecutivo y el Legislativo al definir los derroteros de la economía durante la vigencia de aquél [...]. El Plan, entonces, no es el resultado de trabajos técnicos y económicos elaborados en el interior del Gobierno, ni tampoco el de las deliberaciones que tienen lugar en las cámaras, sino

6 Cuatro (4) alcaldes y cinco (5) gobernadores como representantes de las entidades territoriales; cuatro (4) representantes del sector económico (gremios industriales, productores agrarios, comerciantes, entidades financieras y aseguradoras, microempresarios); cuatro (4) representantes del sector social (gremios de profesionales, campesinos, empleados, obreros, trabajadores independientes e informales); dos (2) representantes del sector educación (agremiaciones de universidades, instituciones de educación primaria y secundaria de carácter público y privado, estudiantes de universitarios y organizaciones cuyo objeto sea el desarrollo científico, técnico o cultural); un representante del sector ecológico (organizaciones cuyo objeto sea la protección y defensa de los recursos naturales y el medio ambiente); un representante del sector comunitario (asociaciones comunitarias); un representante de los indígenas; un representante de comunidades afro-descendientes; un representante de comunidades isleñas raizales del archipiélago de San Andrés y Providencia y dos mujeres representantes de organizaciones no gubernamentales. 
que incorpora, por mandato constitucional, el concepto y el análisis de un foro deliberante y libre que representa a los asociados, cuyo interés en el proceso de planeación es incontrovertible en cuando los afecta de modo directo" (Corte Constitucional, 1996).

No obstante, esta visión normativa que constituye el CNP en un mecanismo eficaz de colaboración y dinamización de las relaciones entre el gobierno colombiano y actores económicos y sociales para la deliberación, planeación y coproducción de estrategias de desarrollo, dista mucho en realidad de ser un espacio efectivo de participación.

En primer lugar, desde su concepción constitucional, el CNP careció de carácter vinculante y decisorio. Los acuerdos alcanzados en el marco del Consejo entre el gobierno y representantes del sector empresarial y social no son acogidos de manera obligatoria en las metas y programas estratégicos del Plan Nacional de Desarro11 o o, en su defecto, en los planes de desarrollo departamentales y municipales. De esta forma, el diseño normativo le otorgó un alcance meramente formal en los procesos de definición de estrategias de desarrollo.

La asociación entre el gobierno y actores no estatales en el marco del CNP se sostiene en la labor de este último de emitir un concepto sobre la propuesta de desarrollo de aquél, no en un trabajo colaborativo y estrecho de coproducción de estrategias de desarrollo y diseño de políticas. De aquí, que la relación de armonía y colaboración entre el Consejo y la entidad que representa al gobierno, el DNP, esté altamente condicionada por el nivel de aprobación o crítica que los demás sectores representados tengan sobre la propuesta oficial (Puentes y Gonzáles, 2011).

Esto ha tenido dos efectos importantes en el proceso de institucionalización del CNP: el primero, la permanente discontinuidad que ha adolecido y la alta politización de su labor. Esto lo explica, en gran medida, el hecho de que el CNP y los consejos territoriales dependan para su funcionamiento de los recursos que la oficina de planeación nacional decida otorgarles.

De aquí, que una postura crítica o una desviación fundamental del proyecto de desarrollo oficial pueda implicar que el DNP retire el apoyo financiero y logístico a Consejo, estrangulándolo en su funcionamiento (Puentes y Gonzáles, 2011). Así, el CNP carece de otra dimensión fundamental en el establecimiento de asociación eficaz y operativa entre actores públicos y privados, es decir, la relativa al nivel de vinculación financiera que exista entre ellos.

De otro lado, el alto nivel de politización del Consejo se refleja también en los procesos de designación y elección de los consejeros y consejeras, en tanto la administración ha permeado los procesos de conformación de las ternas. Esto, ha promovido la constitución de agendas de negociación paralelas a la agenda institucional en las cuales imperan prácticas clientelistas, lógicas rentistas y corrupción, que desvirtúan la naturaleza que la constitución le pretendió otorgar a estos espacios de confluencia social.

A nivel territorial se identifica en mayor medida la alta politización de los Consejos que resulta de la lógica de cooptación por parte del poder ejecutivo. Las autoridades máximas a nivel regional, se resisten a comprender las funciones de los consejos optando por anular su rol cuando se constituyen en un obstáculo para su gestión o usándolo como escenario de legitimación. Por lo anterior, el rol de los consejos territoriales ha oscilado entre una fuerte oposición a las administraciones locales o legitimadores ad hoc de sus acciones. 
Otro elemento que explica la falta de autonomía del Consejo y su incapacidad de servir de foro para la confluencia de diferentes concepciones de desarrollo, ha sido, en palabras de Clemente Forero, ${ }^{7}$ "el déficit de competencias que presentan los miembros para discutir de manera macro sobre el futuro de la sociedad, sobre el rol que debía jugar el Estado en la conducción de la economía y sobre la visión natural que un ejercicio de esta naturaleza demanda para cuestionar el proyecto del Plan de Desarrollo y coproducir con el Gobierno ajustes y estrategias" (Puentes y Gonzáles, 2011: 211).

Dentro de los perfiles que representan los actores no estatales, se encuentran quienes carecen de experiencia y formación en procesos de planeación integral y dirigen su participación a denunciar las consecuencias de estar alejados de los centros de decisión del país y la apatía del Gobierno Nacional. Así mismo, están quienes carecen de una visión global del plan y su participación en el foro de CNP es instrumental, dirigiendo sus aportes a mejorar sus márgenes de negociación con el gobierno y procurar intereses segmentados.

Este último elemento, el conocimiento parcial que tienen los delegados de los diferentes sectores del plan de desarrollo, ha impedido aproximarse a la visión constitucional del CNP de servir de espacio de deliberación, análisis, coproducción y confrontación del proyecto de desarrollo del gobierno por parte de la sociedad civil, antes de someterlo a consideración del Congreso. Por el contrario, el paso del plan de desarrollo por el CNP es, en la práctica, meramente procesal y sirve como legitimador de la propuesta oficial, en tanto la presenta como una obra de construcción colectiva sin que existan, en realidad, aportes sustanciales por parte del Consejo.

Así, entonces, la efectividad del CNP se ha visto socavada en tanto han confluido en su foro prácticas que se alejan de su concepción constitucional, como instancia de colaboración y concurrencia de diferentes actores de la sociedad en la definición de cursos de acción dirigidos al desarrollo económico y la transformación social del país.

De aquí, que, pese a que el CNP es una instancia de diálogo formal y estructurada entre el gobierno y actores no gubernamentales, su institucionalización es altamente débil. Esto, en tanto ha fluctuado entre períodos de cooptación por parte del ejecutivo en todos los niveles, estando al servicio del gobierno y su legitimación, y períodos en los cuales se resiste a esta lógica y se configura como una fuerza de oposición. En ambos casos altamente politizado.

Estas lógicas de cooptación y oposición se explican, en gran medida, desde las propias debilidades de las instituciones del Estado colombiano. El CNP, al igual que otras instancias de coordinación entre el gobierno y actores no gubernamentales en procesos de decisión pública y construcción de políticas, ha sido interrumpido y rediseñado en cada gobierno. Las limitaciones a la hora de sentar las bases de una estrategia de Estado con visión de largo plazo han debilitado, a su vez, su madurez institucional.

Durante la administración del ex presidente Álvaro Uribe Vélez, la función del CNP de servir de foro de acuerdo entre el gobierno y actores no gubernamentales fue, esencialmente, anulada. La administración Uribe, incorporó los llamados consejos comunales, ${ }^{8}$ como mecanismo para acercar la sociedad civil al poder central,

Presidente del Consejo Nacional de Planeación (CNP) en el período 1994-1999.

Los Consejos Comunales de Gobierno son, en esencia, una herramienta de gestión pública incorporada por el ex presidente Álvaro Uribe Vélez y replicada por muchos alcaldes y gobernadores del país y estudiada y adaptada por otros gobiernos latinoamericanos. Consisten en espacios de diálogo y participación ciudadana incorporados por el ex presidente cuando se desempeñó como Gobernador de Antioquia entre 1995 y 1997 y 
los cuales consistieron en reuniones semanales localizadas en diferentes partes del país donde la ciudadanía y representantes de diferentes sectores, expresaban sus necesidades concretas y denuncias directamente al jefe de gobierno y a su gabinete de ministros.

Esta práctica administrativa le restó importancia al CNP y particularmente, a los Consejos Territoriales de Planeación (CTP), en tanto desestimularon la participación de los representantes de diferentes gremios en los Consejos, toda vez que no veían reflejados sus aportes ni los acuerdos alcanzados en los planes de desarrollo y si obtenían respuestas a través de los consejos comunales sin necesidad de incurrir en costos de participación.

Así las cosas, el CNP, como instancia de coordinación y colaboración entre actores públicos y privados, ha estado desprovista de fuerza e institucionalidad.

Pese a que el ordenamiento y el mecanismo de planificación en Colombia incorpora formalmente la asociación entre el gobierno y actores no gubernamentales en los procesos de decisión y elaboración de estrategias de desarrollo, la participación de actores distintos al gobierno no ha trascendido más allá de una retórica de acción colectiva y construcción de concesos, en la práctica, inoperantes.

Si bien el CNP, representa la incorporación de un proceso que tiene como eje la participación de actores privados, económicos y sociales en la coproducción de estrategias de desarrollo y políticas públicas, no es posible afirmar que se constituya en un espacio legítimo de interacción y coordinación entre los distintos actores.

Así, entonces, el esquema de trabajo entre el gobierno y actores no estatales en el marco del CNP está muy alejado de constituir un centro de decisión sustentado en una lógica de colaboración y mucho menos expresa un nuevo modelo de dirección de la sociedad colombiana. En la práctica, el CNP es instancia frágil institucionalmente, carente de organización, corresponsabilidades y de vínculos financieros, desprovista de carácter vinculante, tratada como un trámite y altamente politizada, puesta al servicio del gobierno o en oposición a él.

\subsection{Revisión crítica del CNP desde la perspectiva de la gobernanza moderna}

De acuerdo con la visión constitucional, el CNP pretendía constituirse en la instancia central de colaboración público - privada que reorientara el modelo de decisión pública tradicional de Colombia, altamente jerarquizado y centralista. La aspiración constitucional era generar un cambio gubernativo, sustentado en la reconfiguración del modelo de dirección política del país, que hasta ahora, había suprimido posiciones distintas a la de la élite bogotana y propiciado el origen de organizaciones para-institucionales en diferentes regiones del país, que reclamaban ser tenidas en cuenta.

posteriormente, a nivel nacional bajo sus dos mandatos como presidente. Los Consejos Comunales de Gobierno cumplieron tres funciones básicas: la primera, alinear políticas del Gobierno con las necesidades ciudadanas, como fin último de la participación ciudadana y de un Estado comunitario. La segunda función que cumplen los consejos comunales es incrementar la coordinación interinstitucional entre los niveles nacional, regional y local. Por último, los consejos comunales de gobierno son un espacio de rendición de cuentas permanente de todas las entidades de Gobierno sobre los temas críticos de cada región o de cada sector. En Colombia, se realizaron 236 consejos comunales en todo el territorio nacional, de los cuales $75 \%$ correspondieron a consejos regionales y $25 \%$ a consejos sectoriales sobre temas como educación, infraestructura y vías, desastres naturales, salud, entre otros. 
Esto, sumado a que el gobierno colombiano no acreditaba suficientes capacidades directivas ni calidad institucional, motivó la reorientación, al menos desde lo formal, del principio de dirección política hacia un modelo sustentado en la coordinación con los demás actores del marco democrático. Sin embargo, fuera de su concepción constitucional, el CNP no ha logrado expandir la esfera de deliberación de los asuntos públicos en Colombia.

Pese a que la gobernanza moderna no fue el marco de referencia explícito de la incorporación constitucional de actores no gubernamentales en la esfera de deliberación de los asuntos públicos en Colombia, su perspectiva reconoce un cambio en el modo tradicional de gobernar y dirigir la sociedad. En palabras de Aguilar y Bustelo, la nueva gobernanza "destaca formas de diálogo y negociación que practican los actores públicos y privados para (...) llegar a acuerdos básicos que serán el marco de referencia para elaborar las políticas" (Aguilar y Bustelo, 2010: 8).

De aquí, que esta perspectiva permita establecer si la constitución y prácticas del CNP representan realmente un cambio en la orientación del modo de gobernar del país, hacia uno más descentralizado, horizontal, coordinado e interdependiente entre los diferentes actores de la sociedad y sus recursos.

De acuerdo con Aguilar y Bustelo (2010: 14), para evaluar en qué medida una instancia o práctica de gobernanza acredita carácter público, legitimidad y eficacia, debe determinarse si realmente existe bajo un modelo de gobernanza, públicamente afirmada y técnicamente robusta.

Como se mencionó anteriormente, la visión constitucional fue otorgar al CNP la labor de expandir el espacio de deliberación pública que reclamaba la sociedad colombiana y reorientar el modo de dirección tradicional, altamente jerárquico y concentrado en la élite bogotana. No obstante, el posterior desarrollo normativo restringió su validez pública e institucional limitando el carácter vinculante de las decisiones que fueran tomadas en el marco de esta instancia de confluencia y coordinación entre el gobierno y actores no estatales.

Es importante aclarar que, desde esta perspectiva, el gobierno continúa siendo el actor central y el único capaz de otorgar carácter y dar forma pública a las decisiones y estrategias coproducidas en estas instancias de asociación con actores privados; sin embargo, la validez política está también determinada por la aceptación de los demás actores sociales acerca de su contenido y de la forma cómo se determinan, implicando necesariamente la participación de actores no gubernamentales.

Así las cosas, el CNP fue desprovisto desde su desarrollo normativo de fuerza decisoria, suprimiendo desde su diseño uno de los elementos centrales que supone un modelo de nueva gobernanza. Las estrategias y propuestas desarrolladas sobre pautas de coordinación y consenso en el marco del CNP no son vinculantes ni ascienden necesariamente a las propuestas finales de desarrollo que se presentan ante el Congreso, pese a que son de obligatoria observancia.

El diseño de los mecanismos y canales formales de participación es otro elemento que cuestiona la validez política del CNP como modelo de gobernanza en Colombia. La designación de consejeros y consejeras en todos los niveles -nacional y territoriales facultad exclusiva del presidente de la República, lo cual ha desvirtuado y politizado la labor del Consejo, en tanto los procesos de participación y exclusión de actores sociales y económicos son fácilmente capturados por los intereses del gobierno central. Esta invalidez institucional proviene de las debilidades mismas de las instituciones del Estado y estructurales del sistema democrático en el país. 
Así mismo, el proceso de designación de consejeros ha conducido, en muchos casos, a que los actores privados y organizaciones sociales consideren ilegítimos sus representantes ante el Consejo. Así, entonces, la calidad de la representación y la transparencia en el proceso de designación han erosionado la institucionalidad y el consenso alrededor del CNP como instancia de acción colectiva y con ello, los acuerdos alcanzados y el mismo Consejo han perdido fuerza.

Desde la perspectiva de la calidad técnica, haciendo referencia a la capacidad del CNP de coordinar los recursos -informativos, financieros, tecnológicos, organizativos- de los diferentes actores y proponer estrategias y líneas de intervención frente a los dilemas de desarrollo del país, el CNP tiene importantes limitaciones.

Esto se explica, parcialmente, por las deficientes competencias técnicas y las carencias formativas en procesos de deliberación de los consejeros designados, especialmente en los niveles territoriales del Consejo (CTP). De aquí, que la efectividad del Consejo se ha visto altamente socavada por el bajo grado de calidad técnica de las estrategias consensuadas.

Así, también, el CNP no ha acreditado calidad administrativa ni madurez institucional para coordinar la acción colectiva y establecer mecanismos que equilibren las voces de los actores representados, dada la asimetría en las relaciones de poder y los recursos que detentan los diferentes actores del marco democrático. Esto ha propiciado que el foro deliberativo esté altamente segmentado, sea fácilmente capturado por los intereses de sectores cuya capacidad de movilización es mayor y se distorsione su fin público.

Si bien la nueva gobernanza supone que la coordinación del gobierno con los demás actores de la sociedad le otorga mayor capacidad y eficacia directiva, el CNP en tanto foro central de coordinación, no ha sido provisto de capacidad institucional ni técnica para integrar los diferentes recursos y capacidades de la sociedad colombiana -el poder político, normativo y coactivo del gobierno y los recursos informativos, financieros, tecnológicos y organizativos del sector económico y civil- y mucho menos, reorientar el modelo de dirección.

A pesar que la realidad política de Colombia, las debilidades de su sistema democrático, la persistencia de actores políticos al margen de la ley y las propias deficiencias de sus instituciones, reclaman enérgicamente la necesidad de integrar actores tradicionalmente excluidos, recursos y capacidades en los procesos de decisión pública, la inoperancia del CNP refleja la resistencia del gobierno colombiano a coordinarse con la sociedad.

Dicho de otra forma, la invalidez pública e institucional y técnica del CNP, pese a su pertinencia social, se explica por la rigidez de la estructura gubernativa y gerencial del país, por la resistencia del gobierno a perder relativa autonomía, centralidad y control y por sus limitaciones a la hora de liderar la acción colectiva y coordinar el conjunto de actores del marco democrático.

La desagregación social y la fragmentación del Estado colombiano es la causa estructural y transversal a todos los conflictos y limitaciones del país. Transitar hacia un modo de dirección política que privilegie la coordinación y colaboración del gobierno con actores no estatales no sólo proviene de las presiones que ejerce el proceso democrático sobre el espacio de deliberación pública en Colombia, sino es reclamado como estrategia para la reconstrucción nacional.

Sin embargo, la consolidación del CNP como el espacio central en el cual el gobierno se coordina con los demás actores sociales para definir los cursos estratégicos que debe seguir el país, encuentra restricciones para su consolidación. 
De otro lado, si bien la gobernanza moderna ofrece herramientas explicativas para aproximarse a los nuevos modelos de gobierno y dirección que demanda las sociedades contemporáneas, se identifican en el análisis del CNP desde esta perspectiva una serie de imprecisiones y tensiones teóricas en el entorno social y político-institucional colombiano.

En primera medida, la perspectiva teórica de la gobernanza se sustenta en una visión abstracta del Estado, que supone que la concentración del poder en manos del Estado se produce de manera natural y espontánea. Sin embargo, en Colombia, el poder y la política no residen exclusiva ni plenamente en el Estado. Esta visión teórica no contempla, entonces, un contexto de presencia diferenciada del Estado y en que confluyan otros actores al margen de las instituciones que reclamen ser reguladores y centro del poder político, como ocurre en el caso colombiano.

Así mismo, la gobernanza moderna supone la existencia de una sociedad civil fuerte, funcionalmente diferenciada y bien organizada, donde no exista desigualdad social (Mayntz, 2001). En este punto, la sociedad civil y la ciudadanía también presenta connotaciones diferenciadas en Colombia, en tanto coexisten lógicas institucionales que promueven la diferenciación funcional y organización de la sociedad civil con lógicas clientelistas donde las relaciones entre ciudadanos y gobernantes se sustentan en lealtades transaccionales, subordinación y cooptación.

En este orden de ideas, las restricciones que presenta la consolidación de la labor del CNP como un proceso de gobernanza en el país, se explican desde las propias debilidades del marco institucional y valorativo del sistema democrático colombiano.

\section{Conclusiones}

En Colombia, coexisten varios tipos de diálogos y esquemas de trabajo conjunto entre el gobierno y actores no gubernamentales con diferentes niveles de institucionalización.

A partir de la década de 1960, se introdujo el mecanismo de planificación como tarea de gobierno, el cual fue el marco original de diálogo entre las entidades de gobierno y los principales actores económicos y organizaciones sociales. A partir de aquí, han existido un sinnúmero de iniciativas encaminadas a crear sinergias y mecanismos de coordinación entre los diferentes actores del marco democrático, orientados a soportar los procesos de decisión pública y dirección de la sociedad, tradicionalmente jerárquicos y centralizados.

En una segunda línea de trabajo conjunto, se vincula al sector privado empresarial y a las organizaciones sociales en la gestión y ejecución de políticas públicas. Aquí, se han obtenido resultados positivos en términos generales. El sector privado empresarial y las organizaciones sociales especializadas, han operado con mayor competencia la prestación de servicios de carácter público, sirviendo de palancas de desarrollo de diferentes sectores en el país, particularmente, en la construcción, mantenimiento y operación de infraestructura productiva y social.

Sin embargo, este tipo de alianzas no necesariamente contienen implícita la noción de colaboración, aunque la favorezcan, ni la disposición del gobierno a compartir lo que ha sido de su exclusivo dominio y mucho menos, han implicado un proceso de descentralización de la dirección de la sociedad en Colombia. 
Las instancias de colaboración público-privada dispuestas para asegurar espacios de concertación y coordinación del gobierno con actores privados en procesos de decisión y planificación han tenido un proceso evolutivo diferente al de aquellas que se vinculan únicamente con la fase de ejecución de las políticas y la externalización de funciones públicas.

Si bien, los espacios de diálogo entre el gobierno y otros sectores han estimulado el interés por construir una visión colectiva y una estrategia nacional a largo plazo, el proceso de institucionalización y autonomía de dichas instancias de decisión y planificación coordinada han adolecido de discontinuidad y son, en su mayoría, inoperantes y altamente politizadas, oscilando entre lógicas de cooptación y oposición.

Las limitaciones a la hora de consolidar los imperativos de la colaboración público-privada en Colombia se entienden desde las propias debilidades de las instituciones y la dificultad de las fuerzas políticas para construir consenso. Es el caso del Consejo Nacional de Planeación, el cual se introdujo por mandato constitucional con el objetivo de corregir las desviaciones del modelo de dirección política tradicional que había coartado visiones políticas distintas a las impuestas por la élite política del centro del país.

Sin embargo, su institucionalización es altamente débil y está desprovisto de fuerza. El CNP ha fluctuado entre períodos de cooptación por parte del ejecutivo en todos los niveles, estando al servicio del gobierno y su legitimación, y períodos en los cuales se resiste a esta lógica y se configura como una fuerza de oposición. En ambos casos altamente politizado.

Si bien, el mandato constitucional otorgó al CNP la responsabilidad de concentrar y coordinar al gobierno con los demás actores sociales, económicos y civiles, bajo el mecanismo de planificación, con la intención de fortalecer el proceso democrático, superar la crisis institucional y soportar las decisiones públicas; su posterior desarrollo normativo y marco institucional restringió su fuerza y validez pública.

En primer lugar, el CNP fue desprovisto de fuerza vinculante, suprimiendo desde su diseño uno de los elementos centrales que supone una instancia real y eficaz de colaboración público-privada: su carácter decisorio. Las estrategias y propuestas consensuadas en el marco del CNP no son vinculantes ni ascienden necesariamente a las propuestas finales de desarrollo que se presentan ante el Congreso, pese a que son de obligatoria observancia, otorgándosele un alcance meramente formal.

Así también, otro elemento que cuestiona la validez del CNP y refleja su alto grado de politización, son los mecanismos y canales formales de participación. La designación de consejeros y consejeras en todos los niveles -nacional y territoriales facultad exclusiva del presidente de la República, lo cual desvirtúa la labor del Consejo en tanto la designación de representantes y en su caso, exclusión, es fácilmente capturada por los intereses del gobierno central.

De otro lado, el CNP carece de autonomía y vinculación financiera entre sus miembros, dimensión fundamental para el establecimiento de una asociación eficaz y operativa. El Consejo depende para su funcionamiento de la oficina de planeación nacional. De aquí, que una desviación de la propuesta de desarrollo oficial pueda implicar que el DNP retire el apoyo financiero y logístico al Consejo, estrangulándolo en su funcionamiento.

La carencia de autonomía y la invalidez política y técnica del Consejo para servir de foro de confluencia de diferentes concepciones de desarrollo, resulta también del déficit de competencias que presentan los miembros para discutir de manera 
macro sobre el futuro de la sociedad, sobre el rol que debe desempeñar el Estado colombiano en la conducción de la economía y sobre la visión natural que un ejercicio de esta naturaleza demanda para cuestionar el proyecto de desarrollo y coproducir con el gobierno estrategias.

Así, entonces, la consolidación del CNP como instancia de coordinación entre el gobierno y actores económicos y sociales, cuya vocación constitucional fue reorientar el modelo de dirección política del país e intervenir los procesos de decisión pública en todos los niveles, no ha trascendido más allá de una retórica de acción colectiva y construcción de concesos, en la práctica, inoperante.

El CNP es, entonces, una instancia carente de organización, desprovista de fuerza decisoria, de corresponsabilidades y autonomía financiera, tratada como un trámite, altamente politizada, su diseño institucional es frágil y técnicamente es débil. Sin embargo, estas restricciones no son exclusivas del Consejo, en términos generales las instancias de colaboración entre el gobierno y actores no estatales dispuestas para intervenir los procesos de decisión pública no encuentran terreno para su consolidación.

Así las cosas, después de la constituyente de 1991, en la conducción de la sociedad colombiana han proliferado una retórica de instancias, estructuradas y tácitas, que vinculan a los actores no estatales en los procesos de decisión, pero que en la práctica son inoperantes. En términos generales, la fragilidad institucional de estos esquemas de colaboración proviene de las debilidades mismas de las instituciones del Estado y estructurales del sistema democrático en el país.

Desde la perspectiva de la gobernanza moderna, las decisiones públicas y el diseño de políticas no sólo se valoran desde su contenido, sino esencialmente desde la forma como son tomadas y elaboradas, cuya dinámica central es la coordinación entre actores y la construcción de concesos. Los imperativos de la nueva gobernanza suponen, entonces, un nuevo proceso directivo sustentado en una nueva lógica de coordinación y colaboración entre el gobierno y los demás actores sociales, en función de coproducir estrategias y consensuar posiciones de cara al crecimiento económico y desarrollo social.

Sin embargo, en Colombia los esquemas de colaboración y participación de actores no estatales en procesos de decisión pública, parecen ser utopías consagradas en la constitución. El gobierno colombiano se resiste a coordinarse con la sociedad e ignora el potencial de estas instancias de colaboración en la construcción de cohesión y capital social, especialmente en un país donde el poder no ha residido exclusivamente en el Estado y reclama un proceso de reconstrucción nacional.

Es imperativo, entonces, que el gobierno colombiano transcienda en la práctica hacia un modelo de dirección política que reconozca y privilegie la colaboración real con los demás actores del marco democrático e integre sus recursos a su estructura gubernativa.

\section{Bibliografía}

AECID. (2013). Protocolo AECID para la gestión de alianzas público-privadas para el desarrollo (APPD). AECID, Madrid.

Aguilar, L. \& Bustelo, M. (2010). "Gobernanza y evaluación: una relación potencialmente fructífera”, en Revista Gestión y Análisis de Políticas Públicas, $\mathrm{n}^{\circ} 4$, julio - diciembre. 
Aguilar, L. (2005). Gobernanza y justicia. FLACSO, México.

Aguilar, L. (2007). "El aporte de la política pública y la nueva gestión pública a la gobernanza", en XII Congreso Internacional del CLAD sobre la Reforma del Estado y de la Administración Pública, República Dominicana.

Aguilar, L. (2014). La nueva gobernanza pública. Instituto de Investigación en Política Pública y Gobierno, Universidad de Guadalajara, México.

Araya Orellana, J. (2012). "Alianzas público-privadas y participación: un análisis desde la teoría", en XVII Congreso Internacional del CLAD sobre la Reforma del Estado y de la Administración Pública, Colombia.

Banco Mundial, Fundación Corona. (2009). Evaluación del programa nacional de alianzas entre el sector público y privado y las organizaciones ciudadanas en Colombia. Colombia.

Bazerlay, M. (1998). Atravesando la burocracia. Una nueva perspectiva de la administración pública. Fondo de Cultura Económica, México.

Caplan, K. (2003). The purist's partnership: Debunking the terminology of partnerships. Building Partnerships for Development in Water and Sanitation. University of Cambridge, Cambridge.

Casado Cañeque, F. (2007). Alianzas público-privadas para el desarrollo. Fundación Carolina CEALCI, Madrid.

Casado Cañeque, F. (2008). Hacia la concertación público-privada para el desarrollo en América Latina. Fundación Carolina CEALCI, Madrid.

Congreso de la República de Colombia. (2015). Ley 1753 Plan Nacional de Desarrollo 2014-2018. Diario Oficial No. 49.538 de junio de 2015. Colombia.

Constitución Política de Colombia. (1991). Gaceta Constitucional. Colombia.

Departamento Nacional de Planeación. (2009). Alianzas público privadas en el marco de la RED JUNTOS, Colombia.

Departamento Nacional de Planeación. (2015). Decreto 1082 Reglamentario del Sector Administrativo de Planeación Nacional. Diario oficial Colombia.

Devlin, R. \& Moguillansky, G. (2009). Alianzas público-privadas $\square$ para una nueva visión estratégica del desarrollo. Comisión Económica para América Latina y el Caribe (CEPAL), Naciones Unidas.

Foro Económico Mundial. (2003). Public-private partnerships: Meeting in the middle. Washington y Ginebra, The United Nations Foundation and WEF.

Fundación Naciones Unidas y Foro Económico Mundial. (2003). Public-private partnerships: Meeting in the middle, Washington y Ginebra.

Fundación Naciones Unidas. (2005). Understanding public-private partnerships. Nueva York.

Jessop, B. (1998). "The rise of governance and the risks of failure: The case of economic development”, en International Social Science Journal, vol. 50, $\mathrm{n}^{\circ} 155$, pp. 29-45.

Kooiman, J. (2003). Gobernar en gobernanza. La gobernanza hoy: 10 textos de referencia. España: INAP.

Mayntz, R. (2001). "El Estado y la sociedad civil en la gobernanza moderna", en Revista del CLAD, Reforma y Democracia, No. 21.

Olías De Lima Gete, B. (2001). La nueva gestión pública. Prentice Hall, Madrid.

Olías De Lima Gete, B. (2011). "Cara o cruz de la externalización”, en Álvarez, O. (ed.), Crisis y externalización en el sector público: ¿solución o problema? INAP, Madrid. 
Olías De Lima Gete, B \& Del Campo, E. (2008). "Buen gobierno, rendimiento institucional y participación en las democracias contemporáneas", en Sistema: Revista de Ciencias Sociales, $\mathrm{n}^{\circ}$ 203-204.

Puentes, G \& González, C. (2011). "El Sistema Nacional de Planeación: De la utopía a la cooptación", en Revista Equidad y Desarrollo, vol. 16.

Sen, A. (1999). Development as freedom. Oxford University Press, Oxford.

Serrano, C. (2011). Gobernanza para el desarrollo económico territorial en América Latina. Centro Latinoamericano para el Desarrollo Rural, Colombia.

Zurbriggen, C. (2011). "Gobernanza: Una mirada desde América Latina", en Perfiles Latinoamericanos, $\mathrm{n}^{\circ} 38$, pp. 39-63 
